\title{
What does Stat3 do?
}

\author{
David E. Levy and Chien-kuo Lee \\ Department of Pathology and Kaplan Comprehensive Cancer Center, New York University School of Medicine, New York, New York, USA \\ Address correspondence to: David E. Levy, Department of Pathology, New York University School of Medicine, \\ 550 First Avenue, New York, New York 10016, USA. \\ Phone: (212) 263-8192; Fax: (212) 263-8211; E-mail: levyd01@med.nyu.edu.
}

J. Clin. Invest. 109:1143-1148 (2002). DOI:10.1172/JCI200215650.

The Stat protein family was discovered in the course of studies of signaling specificity from IFN receptors (1). The initial finding of a family of related proteins, each activated by a different cytokine receptor, suggested that these proteins would fulfill the requirements predicted for carriers of intracellular signaling information capable of retaining the specificity inherent in cytokine-receptor interactions (2). While studies in cell culture systems belied some of this early promise, the initial findings with genetically manipulated mice, especially using gene-targeting approaches, generally suggested a high degree of specificity for the various Stat proteins in individual signaling pathways. As reviewed elsewhere in this Perspective series, the analysis of mice lacking one or more Stat genes has shown relatively discrete phenotypes, assigning each Stat protein to a relatively specific pathway. Not so with Stat3.

Unlike all other members of the Stat gene family, ablation of Stat3 leads to embryonic lethality (3). This finding, along with evidence of its activation by a wide variety of cytokines, growth factors, and other stimuli $(4,5)$, implied that Stat 3 might be more generally deployed than its relatives and has led to the suggestion that it might represent a primordial Stat protein. Recent data, especially from the analysis of conditional loss of Stat3 protein in adult tissues, confirm that Stat 3 participates in a wide variety of physiological processes and even directs seemingly contradictory responses.

\section{Discovery of Stat3}

Stat3 was first described as a DNA-binding activity from IL-6-stimulated hepatocytes, capable of selectively interacting with an enhancer element in the promoter of acute-phase genes, known as the acute-phase response element (6-9). Molecular definition of this factor demonstrated that the same protein, a close relative of Stat1, is activated by the entire family of IL-6-type cytokines, which signal through gp130 and related receptors $(5,10,11)$. Moreover, at least in cell culture systems, Stat 3 is also activated by such diverse agents as growth factors, oncogenes, and IFNs.

Structurally, Stat3 is similar to other Stat proteins, having a conserved amino-terminus involved in tetramerization, a DNA-binding domain with a sequence specificity for a palindromic IFN- $\boldsymbol{\gamma}$-activated sequence (GAS) element very similar to that of Stat1, an SH2 domain involved in receptor recruitment as well as Stat dimerization, and a carboxy-terminal transactivation domain. As with other Stat proteins, Stat 3 is activated by tyrosine phosphorylation at a single site close to the carboxy-terminus (Y705), as well as by serine phosphorylation at a site within the transactivation domain (S727). Tyrosine phosphorylation in response to cytokine stimulation is mediated by a Janus kinase, most often JAK1 (12), and is required for Stat 3 dimerization, nuclear translocation, and DNA binding. Targeted phosphorylation of Stat 3 appears to follow the general paradigm of receptor recruitment first worked out for Stat 1 activation in response to IFN- $\gamma(13)$, involving a specific interaction between the Stat $3 \mathrm{SH} 2$ domain and a phosphotyrosine on the gp130 cytoplasmic domain within the consensus sequence YxxQ. Serine phosphorylation occurs within a mitogen-activated protein kinase consensus site.

The identity of the serine kinase for Stat 3 is somewhat controversial, most likely because different activation signals lead to serine phosphorylation by any of several kinases, including ERK1, ERK2, p38, JNK, and an $\mathrm{H}-7-$ sensitive kinase (14). Most evidence suggests a positive role for S727 phosphorylation in Stat 3 transcriptional activation, presumably through enhanced recruitment of necessary transcriptional cofactors, as is the case for serine phosphorylation of Stat 1 (15). However, there is also evidence for a negative role for serine phosphorylation, although its underlying mechanism is unclear.

The function of Stat 3 has been extensively studied in cell culture systems. IL-6-type cytokines evoke a number of distinct responses in different cells, including induction of an acute-phase response in hepatoma cells, stimulation of proliferation in B lymphocytes, activation of terminal differentiation and growth arrest in monocytes (11), and maintenance of the pluripotency of embryonic stem cells (16-19). The finding that Stat 3 is involved in all these distinct functions has suggested that Stat 3 is the major signal transducer downstream of gp130-like receptors. Moreover, this conclusion raises the interesting issue of how a single transcription factor can be involved in seemingly contradictory cell responses. The answer to this riddle may be found at least in part in the induction of distinct sets of target genes by 
Stat 3 in different cells (5). For instance, part of the mechanism by which Stat3 stimulates B cell proliferation is through inhibition of apoptosis, a function mediated by induction of the antiapoptotic gene $\mathrm{Bcl}-2$. In contrast, activation of Stat 3 in monocytic cells leads to downregulation of c-myc and c-myb and induction of junB and IRF-1, a pattern of gene regulation consistent with differentiation and growth arrest. Likewise, a different set of Stat3-dependent genes are upregulated in IL-6-stimulated hepatocytes, genes for the secreted proteins of the acute-phase response (20).

Additional signaling systems also appear to rely heavily on Stat3, at least in cell culture systems. For instance, G-CSF receptor signaling during granulopoiesis leads to a striking activation of Stat3. Moreover, the Stat 3 requirement site on the receptor is required for G-CSF-driven proliferation, and expression of dominant negative Stat 3 impairs proliferation (21). HGF activates Stat 3 during the process of tubule outgrowth in epithelial cells (22). IL-10 requires Stat3 activation for its anti-inflammatory properties on macrophages (23). How a common transcription factor activates a distinct gene program dependent on cell type is an area of active investigation.

Stat3, like other Stat proteins (24), has also been implicated in cancer (see Bromberg, this Perspective series, ref. 25; and references therein). Following the seminal discovery that Stat 3 is constitutively phosphorylated in v-Src-transformed cells (26), considerable evidence has accumulated suggesting a critical role for activated Stat3 during malignant transformation. Activated Stat 3 has been observed in a variety of experimental malignancies, and its abrogation by use of dominant negative inhibitors or antisense oligonucleotides has led to reversal of the malignant phenotype. Expression of a constitutively active version of Stat 3 on its own can lead to fibroblast transformation, suggesting that Stat 3 is an oncogene. Moreover, numerous mouse and human malignancies have shown activated Stat3, including many head and neck cancers, mammary carcinomas, multiple myelomas, and other hematological malignancies. In these situations, Stat 3 has been described as mediating largely a survival function, and induction of such antiapoptotic genes as $\mathrm{Bcl}-2$ and $\mathrm{Bcl}-\mathrm{X}$ has been suggested as a target of Stat 3 action.

\section{Stat3 is required for embryogenesis}

The seemingly contradictory responses mediated by Stat 3 in cell culture have been somewhat difficult to resolve, partly because such studies are by their nature indirect. Often, these studies are limited to observing a correlation between Stat3 phosphorylation and cytokine action. More direct evidence has been obtained through the use of receptor mutants incapable of recruiting Stat3, through ectopic expression of potentially trans-dominant mutants of Stat3, and through mimicking cytokine responses with constitutively active versions of Stat 3 . Biological effects of Stat 3 have also been evaluated by targeted gene ablation in transgenic mice. Unlike the results with ablation of other Stat family genes, all of which have produced viable mice with relatively limited phenotypes, ablation of Stat3 led to early embryonic lethality (3). In fact, loss of Stat 3 is lethal even in embryonic stem cells $(18,19)$. Homozygous Stat3-null embryos degenerate rapidly between 6.5 and 7.5 days of embryogenesis, just after blastocyst implantation. Stat3 mRNA is present in both maternal and extraembryonic tissues during early postimplantation stages of murine development. Furthermore, activated Stat3 protein is present from embryonic days 4.5 to 9.5 in decidual swellings of the visceral endoderm (27). Because the visceral endoderm plays an important supportive role during early embryogenesis, fostering metabolic exchange between embryo and placenta, it has been hypothesized that Stat 3 may be involved in a nutritional process that supports the implanted blastocyst. It should be possible to test this hypothesis by tetraploid rescue experiments. Unfortunately, the inviability of Stat3-null embryonic stem cells would greatly complicate this experiment and would require the use of homozygous blastocysts obtained in vivo by intercrossing Stat $3^{+/-}$mice.

Like Stat3, other components of the gp130 signaling system are also required for embryogenesis. For instance, loss of genes for various receptors, including gp130 and leukemia inhibitory factor receptor $\beta$ (LIFR $\beta$ ), leads to embryonic lethality (28), absence of LIF in female mice results in failure of embryo implantation (29), and cardiotrophin-1 (another gp130 ligand) is required to stimulate embryonic motor neuron survival (30). Jak1, which appears to be the essential receptor-associated kinase for gp130 action $(12,31)$, is also essential (32). However, none of these components explains the early embryonic lethality observed in the absence of Stat3: LIF is required only in maternal tissue, not in the embryo proper, while absence of Stat 3 in the embryo is lethal. Loss of gp 130 and loss of LIFR $\beta$, while producing embryonic lethality, do so at a much later stage than that observed with loss of Stat3, showing that there is an essential Stat3-dependent function that is not mediated by any of the known Stat3-activating cytokines or their receptors. Similarly, growth factor receptors capable of activating Stat 3 appear to act at later stages of development than does Stat3, judging by the phenotypes of loss-of-function mutants $(33,34)$. Even Jak1, which appears to be necessary for all gp130related functions, is not required at the early embryonic stage when Stat 3 first acts, since embryos lacking Jak1 survive to term (32). Similarly, c-src, another tyrosine kinase implicated in Stat3 activation $(26,35)$, is not required for embryogenesis (36), and even embryos lacking all three common src family members survive longer than do those lacking Stat3 $(37,38)$. Therefore, the early embryonic process mediated by Stat 3 remains mysterious, as does the signaling pathway that leads to its activation at this early developmental stage.

\section{Pleiotropic requirements for Stat 3 revealed by conditional knockout mice}

Because classical gene targeting methods reveal only the earliest point in mouse development where a gene plays a critical role, it has been necessary to adopt a conditional gene ablation approach to study Stat3-dependent 
processes at late stages in embryogenesis and beyond. This has been achieved by using the Cre-loxP recombination system. Paired loxP sites have been targeted into introns surrounding critical regions of Stat 3 , including the site for JAK-dependent tyrosine phosphorylation and the SH2 domain $(18,20,39)$. Following introduction of Cre recombinase, deletion of the DNA segment flanked by the $\operatorname{lox} P$ sites results in functional inactivation of the Stat 3 gene. This approach allows the targeting of the Stat 3 mutation to any desired adult or embryonic tissue where Cre expression can be induced. To date, this approach has been used to evaluate Stat3 function in skin, thymic epithelium, T cells, granulocytes, mammary gland, liver, nervous system, and bone marrow (Table 1).

\section{Stat3 in skin}

The keratin 5 (K5) promoter has been used to direct Cre expression to epidermal and follicular keratinocytes (40). The resulting mice are viable despite the absence of functional Stat 3 protein in their epidermis. Hair cycle and wound healing processes are severely compromised in the mutant animals, which display sparse hair and spontaneously develop ulcers with age. However, the first hair cycle is essentially normal in the absence of Stat3. Defects become apparent only with the second cycle and grow more pronounced with age. This phenotype is quite distinct from that seen in the absence of EGFR $(33,34)$, suggesting that a different ligand, perhaps HGF, is responsible for activating Stat 3 in the skin. In addition, migration of keratinocytes in response to growth factor stimulation in vitro was impaired, although the cells displayed normal mitogenic responses. Interestingly, induction of genes necessary for cell migration also appeared normal. Therefore, Stat 3 is necessary for a variety of functions in the skin, some of which are dependent on growth factors such as EGF family members and TGF- $\alpha$, ligands known to activate Stat3. Nonetheless, many responses to these ligands remain intact. These results do not fit a simple model of Stat 3 function being restricted to cell growth, differentiation, or survival but rather suggest that Stat 3 is involved in maintenance of postnatal interactions between epithelia and mesenchymal compartments.

\section{Stat 3 in the thymic epithelium}

Keratin 5 is expressed in thymic epithelial cells, so the same mouse strain used to evaluate Stat 3 function in skin has been examined for effects on thymic function (41). Alterations are only observed in adult mice, which develop severe thymic hypoplasia, including loss of thymocytes and of the normal thymic architecture. These changes appear at the time of normal thymic involution, suggesting that Stat 3 may play a role in the timing or extent of this normal aging process. In young mice prior to developing any overt symptoms, the thymus shows hypersensitivity to apoptosis-inducing agents, such as steroids or $\gamma$-irradiation, although isolated thymocytes are no more sensitive than controls. Interestingly, it is the thymocytes that degenerate in the mutant animals, even though they are wild-type for Stat 3 , since the conditional mutation affects only the neighboring epithelial cells. Therefore, while Stat 3 has been proposed to mediate survival functions, in this case, these functions operate in an indirect manner involving the influence of the thymic microenvironment on T cell survival.

Again, these results defy a simple explanation, such as absence of a set of Stat 3 target gene products. Microarray analysis has been applied to identify misregulated genes in the Stat3-deficient thymus. Rather

Table 1

Summary of Stat3-deficiency phenotypes revealed by conditional gene targeting in mice

\begin{tabular}{|c|c|c|c|c|c|}
\hline $\begin{array}{l}\text { Promoter used } \\
\text { to drive Cre }\end{array}$ & Target cells & Phenotypes & Affected functions & Similar phenotypes ${ }^{\mathrm{A}}$ & Reference \\
\hline K5 & Keratinocytes & $\begin{array}{l}\text { Impaired 2nd hair cycle, } \\
\text { wound repair, and keratinocyte migration }\end{array}$ & Migration & Egfr ${ }^{\prime-}, \mathrm{Tgfr}^{\prime /-}, h g f^{\prime-}$ & (39) \\
\hline K5 & Thymic epithelium & $\begin{array}{l}\text { Age-dependent thymic hypoplasia, } \\
\text { hypersensitivity to stress }\end{array}$ & Survival & $\mathrm{CD} 3 \varepsilon^{\mathrm{Tg}}$ & $(40)$ \\
\hline Lck & T lymphocytes & $\begin{array}{l}\text { Impaired IL-6-dependent survival, } \\
\text { impaired IL-2R } \alpha \text { expression }\end{array}$ & Survival/proliferation & Stat $5 a^{-/-}$ & $(38,41)$ \\
\hline Mlys & Monocytes/Neutrophils & $\begin{array}{l}\text { Enhanced inflammatory response, } \\
\text { chronic colitis and Th1 differentiation }\end{array}$ & - & $1110^{-1-}$ & 43 \\
\hline$B / g$ & Mammary epithelium & Delayed mammary involution & Apoptosis & - & 44 \\
\hline$M x$ & Liver & Impaired acute-phase response & Gene expression & $116^{-/-}$ & 20 \\
\hline $\mathrm{Bal}^{\mathrm{B}}$ & Sensory neurons & Enhanced neuronal apoptosis & Survival & Lifr $\beta^{-/-}$, Cntfro $\alpha^{/-}$ & 45 \\
\hline$N f l$ & Motoneurons & $\begin{array}{l}\text { Impaired survival } \\
\text { after nerve damage }\end{array}$ & urvival (cytokine-stimulated) & $C T-1$ & 45 \\
\hline
\end{tabular}

ASimilar phenotypes, found in other genetically manipulated mice, suggest possible Stat3-activating cytokines that may contribute to the biological function indicated. ${ }^{B}$ Recombination and loss of the "floxed" Stat 3 gene occurs predominantly in the brain in a balancer strain of mice, which express the Cre recombinase in a mosaic distribution. Tg, transgenic. 
than a loss of a set of potential Stat 3 target genes, mutant thymi showed aberrant cortical expression of mRNAs for keratin 5 , the growth factor receptor ErbB2/neu, and the nonclassical MHC class II protein $\mathrm{H} 2-\mathrm{O}$, all of which are otherwise expressed predominantly in medullary regions of the thymic epithelium. Since these molecules are presumably downregulated in the cortex during differentiation, some degree of dedifferentiation may have occurred in the absence of epithelial Stat3.

\section{Stat 3 in T cells}

Possible functions of Stat 3 in T cells have been examined by specific gene ablation using Cre driven by the Lck promoter $(39,42)$. Prior experiments, provided little evidence for an important Stat 3 function in T cells, since most $\mathrm{T}$ cell cytokines selectively activate other Stat family members, such as Stat4, Stat5, and Stat6. However, the effects of IL- 6 are pleiotropic, including immunomodulatory functions on $\mathrm{T}$ cells, that include stimulation of T cell survival (11). Given the important role for Stat 3 downstream of the IL- 6 receptor, therefore, it was not surprising that IL-6-mediated T cell survival is impaired in Stat3-deficient T cells (39). Conversely, while it might have been expected that Stat3 would exert its antiapoptotic action through induction of survival genes, it was unexpected to discover that $\mathrm{Bcl}-2$ is induced in the absence of Stat 3 , similar to its induction in wild-type cells. Therefore, an unknown, Bcl-2-independent mechanism must mediate the survival effects of IL-6 in T cells.

Stat3-deficient $\mathrm{T}$ cells also display a reduced proliferative response to IL-2 stimulation but this phenotype cannot be explained by reduced survival (42). Rather, this effect correlates with reduced expression of CD25, the inducible chain of the IL-2 receptor. $C d 25$ is known to be a Stat target gene, but it has been previously shown to be induced by Stat5a following IL-2 stimulation. While Stat 3 is a minor target for IL-2 signaling, these results demonstrate that it is nonetheless a relevant component of the signaling pathway.

\section{Stat 3 in the myeloid lineage}

Stat 3 function has been examined in macrophages and neutrophils by Cre-mediated gene ablation directed by the macrophage lysozyme (Mlys) promoter (43). Mutant mice display enhanced susceptibility to endotoxic shock and develop chronic enterocolitis with age. The phenotype of these animals is reminiscent of that in mice lacking IL-10, including aberrant expression of MHC class II molecules and increased expression of inflammatory cytokines. IL-10 is an anti-inflammatory cytokine that suppresses induction of TNF- $\alpha$ through a Stat3-dependent pathway (23). Therefore, it is tempting to speculate that the phenotype of Stat3ablated macrophages derives from the inability to suppress production of TNF- $\alpha$ once it has been induced during bacterial infection.

The IFN-inducible $M x$ promoter driving Cre recombinase has been used to ablate Stat 3 in hematopoietic progenitor cells to evaluate its role in granulopoiesis (C. Lee et al., unpublished observations). Despite the con- siderable evidence from cell culture and transgenic mouse experiments that Stat 3 would be critical for various aspects of myeloid development, including the action of G-CSF (21), all major myeloid cell types develop in the absence of Stat 3 , and granulocytes proliferate and differentiate in response to G-CSF.

\section{Stat3 in mammary development}

Stat5 is critical for mammary development, due to its role as a signaling target for growth hormone and prolactin. In mice lacking Stat5, mammary lobuloalveolar outgrowth during pregnancy is curtailed and terminal differentiation does not occur, leaving females unable to lactate. Stat 3 phosphorylation occurs coincident with mammary gland involution, and the absence of Stat 3 due to Cre recombinase driven by the $\beta$-lactoglobulin (Blg) promoter delays the onset of involution (44). Mammary gland involution is thought to be triggered by absence of the survival factor IGF-1, which becomes sequestered by binding proteins, such as IGFBP5. Interestingly, expression of IGFBP5, which occurs during involution of control glands, is nearly abolished in mutant glands. However, other markers of apoptosis, such as Bcl-X and Bax, were unaffected, and aberrant expression of other survival molecules may occur as a consequence of the lack of apoptosis, rather than a cause. Therefore, it would appear that Stat 3 is proapoptotic in the involuting mammary gland, possibly through a role in regulating the expression of IGFBP5. This role for Stat 3 would appear to be opposite to the involution-suppressing effects of Stat3 in thymus (41).

\section{Stat3 in the nervous system}

Stat 3 is known to be activated in the brain, particularly in response to ciliary neutrophic factor (CNTF) and leptin. A balancer strain of Cre transgenic mice has been used to examine the role of Stat 3 in vivo by generating mosaic animals with differential gene inactivation in different tissues. Because deletion is particularly effective in the nervous system, Stat 3 is lost in the brain, resulting in perinatal lethality (45). Stat3 is required for the neurotrophic effects of CNTF and LIF on developing sensory neurons in vitro, consistent with the finding that few of these cells survive in vivo. Stat3-deficient neurons are unable to induce Akt phosphorylation in response to CNTF, suggesting a direct link between activation of Stat 3 and of Akt. It is unclear what underlying mechanism would link Akt activation by CNTF through Stat3, although it has been suggested that Stat 3 can function as an adaptor molecule that promotes phosphatidylinositol 3-kinase activation in response to IFN. A similar survival function has been found for Stat 3 in injured motoneurons, by using the neurofilament light chain $(\mathrm{N} f \mathrm{l})$ promoter to drive Cre (46). Motoneurons in these animals, unlike controls that maintain one wild-type Stat3 allele, undergo cell death within weeks of axotomy. These findings are consistent with the requirement of Stat 3 for signaling from gp130-type cytokines, such as CNTF, LIF, or CT-1.

\section{Stat 3 and the acute-phase response}

Induction of the acute-phase response in the liver during inflammation is a classic function of IL-6 (7), and 
inactivation of Stat 3 in the liver led to significant impairment of the acute-phase response (20). Genes induced during the acute phase have been categorized according to their time of induction, their promoter structures, and their dependence on IL-6. In the absence of Stat 3 , genes exclusively regulated through Stat-binding sites are silent, while those dependent on other transcription factors, including CAAT/enhancer binding protein (C/EBP) family members and NF- $\kappa B$, are affected to a lesser extent. These results clearly demonstrate the fundamental importance of Stat 3 to the cytokine-mediated induction of acute-phase response genes in vivo. Stat 3 appears to function as a classical transcription factor during this response, and its requirement is directly related to the presence of Stat-binding sites in the promoters of target sites.

It is interesting that Stat 3 was discovered because of its role in the acute-phase response, and that this is the only capacity in which Stat3 function in vivo can be clearly ascribed to its activity as a transcription factor. Nevertheless, there are also surprises in the liver. The truncated Stat $3 \beta$ protein produced by alternative splicing has not received much attention, because its lack of a transactivation domain implied no role in gene expression. However, recent results from specific ablation of this isoform in transgenic mice indicate that it is an important negative regulator, at least during the acute-phase response to endotoxic shock (47). Stat $3 \beta$ deficient mice recover poorly from endotoxic shock and display significant hyperresponsiveness of a subset of inducible genes in the liver. Therefore, Stat $3 \beta$ may be an important negative regulator of Stat $3 \alpha$-inducible genes. This negative regulation appears to be critical for normal recovery following systemic inflammation.

\section{Physiological importance of Stat3}

When first discovered, Stat 3 appeared to be a straightforward additional member of the growing Stat family that would function in the induction of a limited set of target genes in response to IL- 6 released during inflammation. The list of its possible functions grew through subsequent studies in cell culture, showing that it is activated in a wide variety of signaling systems and mediates a bewildering complexity of responses. While similar confusion relating to other Stat family members has been somewhat laid to rest due to the restricted phenotypes observed in knockout mice, Stat 3 remains something of an enigma. Early embryonic lethality in the absence of Stat 3 suggests an essential role in an early developmental process, but the nature of that process, its physiological activator, and even its site of action (in embryonic or extraembryonic tissue) remain to be defined. In particular, none of the known Stat3 activators can account for this embryonic function.

Despite the clear importance of Stat 3 during very early development, the ablation of Stat 3 in adult tissues leads to surprisingly mild phenotypes. All tissues, including all cells of the hematopoietic system, appear to develop normally, although their responses to particular cytokines are impaired. In some cases, the action of Stat 3 can be ascribed to the induction of a set of important target genes, but in others it may be acting as a repressor (e.g., in thymic epithelium) or as a signaling adaptor without a transcriptional function (e.g., activation of Akt in neurons). Moreover, even in situations where Stat3 appears to function as a transcriptional activator, the biological readout can be proliferation, survival, or apoptosis, depending on the target tissue. Thus, although we possess a wealth of data on Stat 3 functions in various contexts, we are still unable to describe fully what Stat3 does. The finding, discussed in detail by Bromberg in this Perspective series (25), that Stat 3 may be a key player in the pathogenesis of diverse human cancers makes this molecule a prime target for novel therapies and lends greater urgency to answering this complex question.

\section{Acknowledgments}

We thank our colleagues for helpful discussions and apologize to all whose work was not properly cited due to the space constraints of a short review. Work in the authors' laboratory was funded by the NIH, the American Heart Association, and the Mathers Charitable Foundation.

1. Darnell, J.E., Kerr, I.M., and Stark, G.R. 1994. Jak-STAT pathways and transcriptional activation in response to IFNs and other extracellular proteins. Science. 264:1415-1421.

2. Levy, D.E., and Darnell, J.E. 1990. Interferon-dependent transcriptional activation: signal transduction without second messenger involvement? New Biol. 2:923-928.

3. Takeda, K., et al. 1997. Targeted disruption of the mouse Stat 3 gene leads to early embryonic lethality. Proc. Natl. Acad. Sci. USA. 94:3801-3804.

4. Takeda, K., and Akira, S. 2000. STAT family of transcription factors in cytokine-mediated biological responses. Cytokine Growth Factor Rev. 11:199-207.

5. Hirano, T., Ishihara, K., and Hibi, M. 2000. Roles of STAT3 in mediating the cell growth, differentiation and survival signals relayed through the IL-6 family of cytokine receptors. Oncogene. 19:2548-2556.

6. Akira, S., et al. 1994. Molecular cloning of APRF, a novel IFN-stimulated gene factor 3 p91-related transcription factor involved in the gp130mediated signaling pathway. Cell. 77:63-71.

7. Lütticken, C., et al. 1994. Association of transcription factor APRF and protein kinase Jak1 with the interleukin-6 signal transducer gp130. Science. 263:89-92.

8. Zhong, Z., Wen, Z., and Darnell, J.E. 1994. Stat3: a STAT family member activated by tyrosine phosphorylation in response to epidermal growth factor and interleukin-6. Science. 264:95-98.

9. Raz, R., Durbin, J.E., and Levy, D.E. 1994. Acute phase response factor and additional members of the interferon-stimulated gene factor 3 family integrate diverse signals from cytokines, interferons, and growth factors. J. Biol. Chem. 269:24391-24395

10. Taga, T., and Kishimoto, T. 1997. gp130 and the interleukin-6 family of cytokines. Annu. Rev. Immunol. 15:797-819.

11. Heinrich, P.C., Behrmann, I., Müller-Newen, G., Schaper, F., and Graeve, L. 1998. Interleukin-6-type cytokine signalling through the gp130/Jak/STAT pathway1. Biochem. J. 334:297-314.

12. Guschin, D., et al. 1995. A major role for the protein tyrosine kinase JAK1 in the JAK/STAT signal transduction pathway in response to interleukin-6. EMBO J. 14:1421-1429.

13. Greenlund, A.C., Farrar, M.A., Viviano, B.L., and Schreiber, R.D. 1994. Ligand-induced IFN gamma receptor tyrosine phosphorylation couples the receptor to its signal transduction system (p91). EMBO J. 13:1591-1600.

14. Decker, T., and Kovarik, P. 2000. Serine phosphorylation of STATs. Oncogene. 19:2628-2637.

15. Zhang, J.J., et al. 1998. Ser727-dependent recruitment of MCM5 by stat1alpha in IFN-gamma-induced transcriptional activation. EMBOJ. 17:6963-6971.

16. Boeuf, H., Hauss, C., Graeve, F.D., Baran, N., and Kedinger, C. 1997. Leukemia inhibitory factor-dependent transcriptional activation in embryonic stem cells. J. Cell Biol. 138:1207-1217.

17. Niwa, H., Burdon, T., Chambers, I., and Smith, A. 1998. Self-renewal of pluripotent embryonic stem cells is mediated via activation of STAT3. Genes Dev. 12:2048-2060.

18. Raz, R., Lee, C.K., Cannizzaro, L.A., d'Eustachio, P., and Levy, D.E. 1999 Essential role of STAT3 for embryonic stem cell pluripotency. Proc. Natl. Acad. Sci. USA. 96:2846-2851. 
19. Matsuda, T., et al. 1999. STAT3 activation is sufficient to maintain an undifferentiated state of mouse embryonic stem cells. EMBO J. 18:4261-4269.

20. Alonzi, T., et al. 2001. Essential Role of STAT3 in the control of the acutephase response as revealed by inducible gene inactivation in the liver. $\mathrm{Mol}$. Cell. Biol. 21:1621-1632.

21. McLemore, M.L., et al. 2001. STAT-3 activation is required for normal GCSF-dependent proliferation and granulocytic differentiation. Immunity. 14:193-204.

22. Boccaccio, C., et al. 1998. Induction of epithelial tubules by growth factor HGF depends on the STAT pathway. Nature. 391:285-288.

23. Riley, J.K., Takeda, K., Akira, S., and Schreiber, R.D. 1999. Interleukin-10 receptor signaling through the JAK-STAT pathway. Requirement for two distinct receptor-derived signals for anti-inflammatory action. J. Biol. Chem. 274:16513-16521.

24. Levy, D.E., and Gilliland, D.G. 2000. Divergent roles of STAT1 and STAT5 in malignancy as revealed by gene disruptions in mice. Oncogene. 19:2505-2510

25. Bromberg, J. 2002. Stat proteins and oncogenesis. J. Clin. Invest. 109:1139-1142. DOI:10.1172/JCI200215650.

26. Yu, C.-L., et al. 1995. Enhanced DNA-binding activity of a Stat3-related protein in cells transformed by the Src oncoprotein. Science. 269:81-83.

27. Duncan, S.A., Zhong, Z., Wen, Z., and Darnell, J.E. 1997. STAT signaling is active during early mammalian development. Dev. Dyn. 208:190-198.

28. Yoshida, K., et al. 1996. Targeted disruption of gp130, a common signal transducer for the interleukin 6 family of cytokines, leads to myocardial and hematological disorders. Proc. Natl. Acad. Sci. USA. 93:407-411.

29. Stewart, C.L., et al. 1992. Blastocyst implantation depends on maternal expression of leukaemia inhibitory factor. Nature. 359:76-79.

30. Oppenheim, R.W., et al. 2001. Cardiotrophin-1, a muscle-derived cytokine, is required for the survival of subpopulations of developing motoneurons. J. Neurosci. 21:1283-1291.

31. Ernst, M., Oates, A., and Dunn, A.R. 1996. Gp130-mediated signal transduction in embryonic stem cells involves activation of Jak and Ras/mitogen-activated protein kinase pathways. J. Biol. Chem. 271:30136-30143.

32. Rodig, S.J., et al. 1998. Disruption of the Jak1 gene demonstrates obligatory and nonredundant roles of the Jaks in cytokine-induced biologic responses. Cell. 93:373-383.

33. Sibilia, M., and Wagner, E.F. 1995. Strain-dependent epithelial defects in mice lacking the EGF receptor. Science. 269:234-238.
34. Threadgill, D.W., et al. 1995. Targeted disruption of mouse EGF receptor: effect of genetic background on mutant phenotype. Science. 269:230-234.

35. Cirri, P., et al. 1997. c-Src activates both STAT1 and STAT3 in PDGF-stimulated NIH3T3 cells. Biochem. Biophys. Res. Commun. 239:493-497.

36. Soriano, P., Montgomery, C., Geske, R., and Bradley, A. 1991. Targeted disruption of the c-src proto-oncogene leads to osteopetrosis in mice. Cell. 64:693-702.

37. Klinghoffer, R.A., Sachsenmaier, C., Cooper, J.A., and Soriano, P. 1999. Src family kinases are required for integrin but not PDGFR signal transduction. EMBO J. 18:2459-2471.

38. Stein, P.L., Vogel, H., and Soriano, P. 1994. Combined deficiencies of Src, Fyn, and Yes tyrosine kinases in mutant mice. Genes Dev. 8:1999-2007.

39. Takeda, K., et al. 1998. Stat3 activation is responsible for IL-6-dependent $\mathrm{T}$ cell proliferation through preventing apoptosis: generation and characterization of $\mathrm{T}$ cell- specific Stat3-deficient mice. J. Immunol. 161:4652-4660.

40. Sano, S., et al. 1999. Keratinocyte-specific ablation of Stat3 exhibits impaired skin remodeling, but does not affect skin morphogenesis. EMBO J. 18:4657-4668.

41. Sano, S., et al. 2001. Stat3 in thymic epithelial cells is essential for postnatal maintenance of thymic architecture and thymocyte survival. Immunity. 15:261-273.

42. Akaishi, H., et al. 1998. Defective IL-2-mediated IL-2 receptor alpha chain expression in Stat3-defiient T lymphocytes. Int. Immunol. 10:1747-1751.

43. Takeda, K., et al. 1999. Enhanced Th1 activity and development of chronic enterocolitis in mice devoid of Stat 3 in macrophages and neutrophils. Immunity. 10:39-49.

44. Chapman, R.S., et al. 1999. Suppression of epithelial apoptosis and delayed mammary gland involution in mice with a conditional knockout of Stat3. Genes Dev. 13:2604-2616.

45. Alonzi, T., et al. 2001. Role of STAT3 and PI 3-kinase/Akt in mediating the survival actions of cytokines on sensory neurons. Mol. Cell. Neurosit. 18:270-282.

46. Schweizer, U., et al. 2002. Conditional gene ablation of Stat 3 reveals differential signaling requirements for survival of motoneurons during development and after nerve injury in the adult. J. Cell Biol. 156:287-297.

47. Yoo, J.Y., Huso, D.L., Nathans, D., and Desiderio, S. 2002. Specific ablation of Stat3beta distorts the pattern of Stat3-responsive gene expression and impairs recovery from endotoxic shock. Cell. 108:331-344. 